\title{
Airway Response to Exercise by Forced Oscillations in Asthmatic Children
}

\author{
CYRIL SCHWEITZER, IMEN BEN ABDELKRIM, HÉLÈNE FERRY, FRÉDÉRIQUE WERTS, SILVIA VARECHOVA, \\ AND FRANÇOIS MARCHAL
}

\author{
Service d'explorations fonctionnelles pédiatriques [C.S., I.B.A., H.F., F.W., S.V., F.M.], Hôpital d'enfants, Vandoeuvre F-54500, France; \\ Laboratoire de Physiologie [C.S., F.M.], EA 3450, Université Henri Poincaré, Vandoeuvre F-54500, France
}

\begin{abstract}
Forced expiratory volume in $1 \mathrm{~s}\left(\mathrm{FEV}_{1}\right)$ detection of exercise-induced bronchoconstriction (EIB) to identify asthma has good specificity but rather low sensitivity. The aim was to test whether sensitivity may be improved by measuring respiratory resistance (RRS) by the forced oscillation technique (FOT). Forty-seven asthmatic and 50 control children (5-12 y) were studied before and after running $6 \mathrm{~min}$ on a treadmill. RRS in inspiration (RRsi) and expiration (RRse), $\mathrm{FEV}_{1}$ and RRsi response to a deep inhalation (DI) were measured before and after exercise. In asthmatics versus controls, exercise induced significantly larger increases in Rrsi $(p<$ $0.001)$ and larger decreases in $\mathrm{FEV}_{1}(p=0.004)$. Asthmatics but not controls showed more bronchodilation by DI after exercise ( $p=$ 0.02 ). At specificity $>0.90$, sensitivity was 0.53 with $25 \%$ increase RRsi and 0.45 with $27 \%$ increase RRse or $5 \%$ decrease $\mathrm{FEV}_{1}$. It is concluded that the FOT improves sensitivity of exercise challenge, and the Rrsi response to DI may prove useful in identifying the mechanism of airway obstruction. (Pediatr Res 68: 537-541, 2010)
\end{abstract}

$\mathrm{E}^{x}$ xercise-induced bronchoconstriction (EIB) is closely linked to airway inflammation and unlikely to develop in healthy children (1), so that detecting airway hyperresponsiveness to exercise in the lung function laboratory is considered highly specific of asthma, i.e. it is associated with low rate of false-positive responses. A limitation is the rather low sensitivity of the test $(2,3)$. EIB has been identified in primary school children by changes in forced expiratory volume in $1 \mathrm{~s}$ $\left(\mathrm{FEV}_{1}\right)$ or peak expiratory flow, and decision levels were mostly based on the former parameter (3). Respiratory resistance (RRS) measured by the forced oscillation technique (FOT) offers an alternative assessment of airway caliber, the time variations of which may be characterized for instance using a single excitation frequency (4). Computing RRs separately in inspiration and expiration (RRsi and RRse, respectively) rather than over the whole breathing cycle may be of interest because the upper airways, which may represent a confounding factor in assessing the intrathoracic airways, are known to contribute differently to airway mechanics in inspiration and expiration $(5,6)$. Furthermore, the RRS change in relation to volume history, more specifically the bronchomotor alteration that follows a deep inhalation (DI), has potential

Received January 14, 2010; accepted July 27, 2010.

Correspondence: François Marchal, M.D., Service d'explorations fonctionnelles pédiatriques, Hôpital d'enfants, Allée du Morvan, F-54500 Vandoeuvre, France; e-mail: f.marchal@chu-nancy.fr

Supported by Grant CPRC from the Centre Hospitalier Universitaire de Nancy and Grant EA 3450 from the Ministère de la Recherche. relevance in identifying the mechanism of EIB (7-9). Indeed, stretching the acutely contracted bronchial smooth muscle promotes bronchial wall relaxation, which in turn could be taken as an indicator of the magnitude of the airway response (10). To the best of our knowledge, a systematic analysis of diagnostic value of single-frequency RRs has not been performed during case-control identification of EIB in the lung function laboratory at school age.

Therefore, the aim of this study was to assess the value of the FOT in identifying EIB in asthmatic children. More specifically, RRSi and RRse and the change in RRS induced by DI were examined with reference to spirometry. Because the exercise test is unlikely to show abnormal results in healthy children, the study focused on comparing parameter sensitivities at specificity $>0.90$.

\section{MATERIALS AND METHODS}

Subjects. Asthmatic children aged 5-12 y were recruited from the local pediatric pulmonology clinic (Hôpital d'enfants, CHU de Nancy, Vandoeuvre, France). Diagnosis of asthma was based on a previous history of typical asthma attacks and positive clinical response to bronchodilators. Treatment was discontinued before the study day: short-acting bronchodilators $>12 \mathrm{~h}$; long-acting bronchodilators $>24 \mathrm{~h}$; and inhaled steroids $>1 \mathrm{wk}$. Age-matched healthy children recruited from local primary schools during the same period of time served as controls. Criteria to enter the control group included a medical history negative for asthma attack, chronic respiratory symptoms, exercise-induced wheezing, dyspnea, chest tightness, and cough. $\mathrm{FEV}_{1}>70 \%$ predicted (11) was required to perform exercise. All subjects were free of respiratory symptoms at the time of the study. Written informed consent was obtained from the children and their parents. The protocol was reviewed and approved by the local committee for human subject protection in biomedical research (CPPRB, CHU de Nancy, France).

RRs measurement. The measuring system (Pulmosfor, SEFAM, Vandoeuvre, France) conformed with recommendations issued by a task force from the European Respiratory Society (12). Briefly, pressure oscillations were applied around the child's head, and measurements were obtained at $12 \mathrm{~Hz}$ with minimal upper airway wall motion (13). The excitation frequency, slightly higher than recommended for the standard input impedance (12), has the further potential of optimizing the signal-to-noise ratio. After the initial familiarization of the child with the equipment and preliminary trials, the acquisition of airflow, tidal volume, and transrespiratory pressure signals was started for 30-40 s. Tidal volume and RRS signals were displayed immediately thereafter, inspected, selected, and stored on disk. RRS oscillation per oscillation was filtered as described previously (14), and the mean for the acquisition period was computed for RRsi and RRse.

\footnotetext{
Abbreviations: DI, deep inhalation; $\Delta \mathbf{R R S}_{\text {DI }}$, change in RRs induced by DI; EIB, exercise-induced bronchoconstriction; $\mathbf{F E V}_{\mathbf{1}}$, forced expiratory volume in $1 \mathrm{~s}$; FOT, forced oscillation technique; FVC, forced vital capacity; RRs, respiratory resistance; RRsi, respiratory resistance in inspiration; RRse, respiratory resistance in expiration
} 


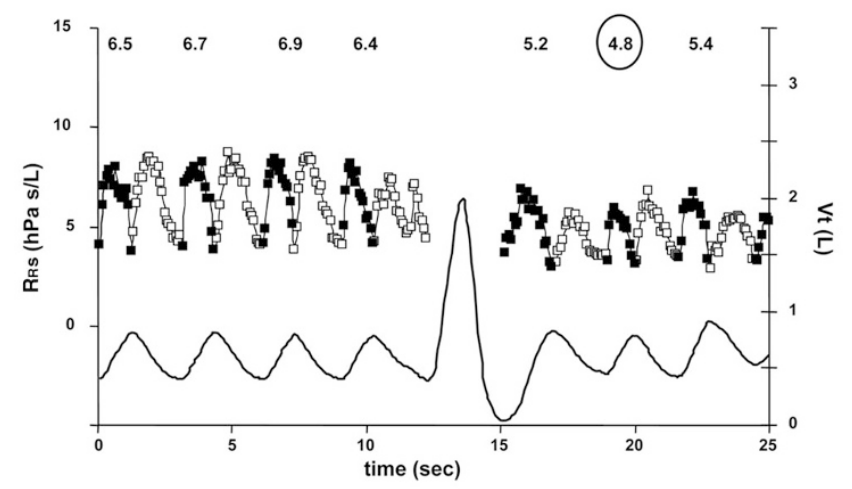

Figure 1. Representative deep inspiration maneuver: Vt, tidal volume, RRsi, and RRse, $\square$. Note typical flow-related RRS variations. RRSi is averaged breath by breath (upper numerical values) and the mean from the four breaths before the DI serves as reference. The lowest RRsi after the DI (second breath here) is taken to compute the bronchomotor response (see Methods).

Effect of DI. After a minimal period of 1 min regular breathing, the child was asked to perform a quick full inspiration and resume normal breathing, while RRS was measured continuously (8). The maneuver was accepted when tidal volume was reasonably regular throughout, the DI volume at least $40 \%$ predicted forced vital capacity (FVC), and the number of validated impedance data sufficiently large to compute a representative time course. Irregular breathing was in fact the primary cause for excessive filtering of these data. Rrsi was averaged breath by breath, and the overall mean from 4 to 5 breaths before the DI served as reference. A representative DI maneuver is illustrated in Figure 1. The bronchomotor effect of DI was eventually computed as the difference between lowest of three post-DI RRSi and the reference $\left(\Delta \mathrm{RRS}_{\mathrm{DI}}\right.$, $\mathrm{hPa} \mathrm{s} / \mathrm{L}$ ). A negative value indicates bronchodilation by DI. The data from a given subject were retained when available at different times of the protocol.

Spirometry. Forced spirometry was performed using an electronic flowmeter with computer animation programs (Masterscope; Erich Jaeger GmbH, Wuertzburg, Germany) as previously described (15). The forced expiratory maneuver was explained to the child, and trials were performed. Forced expiratory maneuvers were repeated until at least two curves displaying early rise to peak flow followed by regular decrease throughout the expiration were obtained, with largest FVC within $10 \%$ of each other. This was usually obtained within five trials. The best curve was selected as the one with highest sum FVC $+\mathrm{FEV}_{1}$

Exercise. Exercise challenge consisted of a 6-min run on a treadmill (h/p/cosmos mercury med 4.0; Nussdorf-Traunstein, Germany) in a climate room, where absolute humidity was kept $<10 \mathrm{mg} / \mathrm{L}(2,16)$. Air temperature and water content were measured daily using a thermohygrometer (Thermometer Hygrometer Delta Ohm, HD 8901 Padua, Italy) and their respective mean $\pm \mathrm{SD}$ values were $13.5 \pm 1.2^{\circ} \mathrm{C}$ and $6.4 \pm 2.1 \mathrm{mg} / \mathrm{L}$. Heart rate was monitored using a heart rate monitor (Polar B1, Helsinki, Finland). The endpoint was an increase in cardiac frequency to at least $80 \%$ of the predicted maximum within the first 2 min and maintaining this value throughout the test by adjusting the treadmill speed between 4 and $10 \mathrm{~km} / \mathrm{h}$ and/or slope between 2 and $8 \%$. The predicted maximal frequency (beat per min) was calculated from the conventional prediction formula as 220 (beat per min) - age (yr).

Protocol. Duplicate RRS, DI maneuver, and spirometry were obtained, in that order, about $10 \mathrm{~min}$ apart at baseline. Exercise was performed. Measurements were repeated as above, 5 and $15 \mathrm{~min}$ after cessation of exercise. The clinical condition, including transcutaneous oxygen saturation (Ohmeda Biox 3700, Louisville, KY), was monitored throughout the testing. When the child exhibited an excessive clinical response $5 \mathrm{~min}$ after exercise, i.e. severe dyspnea and wheezing or decreased air entry and a decrease in oxygen saturation $<95 \%$, usually associated with decrease in $\mathrm{FEV}_{1}>30 \%$ from baseline, the testing was discontinued and salbutamol (Ventoline $10 \mathrm{mg} / \mathrm{mL}$ ) nebulized for $10 \mathrm{~min}$ (Intersurgical Cirrus nebulizer Pediatric Mask, Wokingham, United Kingdom) was given.

Data analysis. A coefficient of repeatability was computed as unsigned difference between baseline measurements and expressed as percentage of corresponding mean. The response to exercise was computed as difference between parameter values 5 or 15 min after exercise and mean baseline and expressed as percentage of the latter. Group means were compared using $t$ test, and a difference was considered statistically significant at $p<0.05$, and corresponding data were expressed as mean \pm SD unless otherwise indicated. The decision level yielding maximal sensitivity at specificity $>0.90$ was
Table 1. Characteristics of the children

\begin{tabular}{|c|c|c|}
\hline & Asthma & Control \\
\hline$n$ & 47 & 50 \\
\hline Gender (boy/girl) & $26 / 21$ & $23 / 27$ \\
\hline Age $(y r)$ & $9(2)^{*}$ & $8(2)$ \\
\hline Height (cm) & $134(11)$ & $132(11)$ \\
\hline Weight (kg) & $32(9)$ & $29(9)$ \\
\hline FVC $(\%)$ & $104(12)$ & $100(12)$ \\
\hline $\mathrm{FEV}_{1}(\%)$ & $101(12)$ & $102(12)$ \\
\hline $\mathrm{FEV}_{1} / \mathrm{FVC}(\%)$ & $88(6) \dagger$ & $92(4)$ \\
\hline Rrsi (hPa s/L) & $6.9(1.9)$ & $7.1(2.2)$ \\
\hline RRse (hPa s/L) & $8.0(2.7)$ & $8.8(3.2)$ \\
\hline
\end{tabular}

* Mean (SD).

$\dagger p=0.001$ vs control.

established using the larger of the two (5 and $15 \mathrm{~min}$ ) responses. Sensitivity was the incidence of positive responses to exercise in the asthma group and specificity the incidence of negative responses in the healthy group. Note the mean RRS (in RRsi or RRse) computed from the whole acquisition period was used to express the bronchomotor response to exercise, whereas the effect of DI was based on a breath by breath computation of RRsi, as shown in Figure 1.

\section{RESULTS}

Forty-seven asthmatic children (21 girls) were recruited. Twenty-six had a history of dyspnea, chest tightness, or cough on exercising. Atopy was diagnosed in 36 by positive skin prick tests and/or increased serum-specific IgE level. Twenty required bronchodilators on demand and 27 inhaled steroids at a daily dose of $100-400 \mu \mathrm{g}(n=19)$ or $>400 \mu \mathrm{g}(n=8)$. Fifty healthy primary school children (27 girls) served as controls (17). There was no significant difference between groups in age, body height, or weight (Table 1).

Baseline lung function. FVC, FEV ${ }_{1}$, RRsi, or RRse were not different between groups, but $\mathrm{FEV}_{1} / \mathrm{FVC}$ was significantly lower in asthma compared with control $(p=0.001$, Table 1$)$. The FEV ${ }_{1}$ coefficients of repeatability were similar between asthma and control for $\mathrm{FEV}_{1}(2.9 \%$ versus $2.6 \%)$, RRsi $(8.8 \%$ versus $10.8 \%$ ), and Rese (11.7\% versus $12.2 \%$ ). However, in asthmatics, RRS repeatability was found to be better in RRsi than RRse $(p=0.02)$.

Responses to exercise. For technical reasons, spirometry could not be obtained at $5 \mathrm{~min}$ in one control subject and RRS at $15 \mathrm{~min}$ in another one. Moreover, two asthmatic children exhibiting clinically significant EIB were nebulized immediately after the 5-min measurement.

The maximal heart rate achieved during exercise was similar between asthma $(180 \pm 1 \mathrm{bpm})$ and control $(178 \pm 2$ bpm). Five min after exercise, the residual increase in minute ventilation from baseline was not different between asthma $(18 \pm 28 \%)$ and control $(14 \pm 23 \%)$, whereas the airway response was significantly larger in asthma by $\operatorname{FEV}_{1}(p=$ $0.004)$, RRsi $(p<0.001)$, and RRse $(p<0.001$, Table 2$)$. Fifteen min after exercise, the airway response was still significantly larger in asthma than control for $\operatorname{FEV}_{1}(p<$ $0.001)$, Rrsi $(p=0.008)$, and Rrse $(p=0.03$, Table 2$)$.

Deep inhalation. The data on response to DI were discarded in 14 asthmatics and 19 controls because of irregular breathing pattern, and in four asthmatics, because the postexercise measurement showed either insufficient DI $(n=2)$ or 
Table 2. Airway response 5 and 15 min after exercise

\begin{tabular}{cccc}
\hline Time & Asthma & Control & \multicolumn{1}{c}{$p$} \\
\hline 5 min $(\%)$ & & & \\
$n$ & 47 & 50 & \\
FEV $_{1}$ & $-5(10)$ & $-0.4(4)^{*}$ & 0.004 \\
RRsi & $30(38)$ & $5(12)$ & $<0.001$ \\
RRse & $25(37)$ & $5(16)$ & $<0.001$ \\
15 min $(\%)$ & & & \\
$n$ & 45 & 50 & \\
FEV & $-2(5)$ & $1(4)$ & $<0.001$ \\
RRsi & $8(27)$ & $-4(13)^{*}$ & 0.008 \\
RRse & $7(27)$ & $-4(18)^{*}$ & 0.03 \\
\hline
\end{tabular}

$* n=49$.

Table 3. Airway response to deep inhalation before and after exercise

\begin{tabular}{lccc}
\hline \multicolumn{1}{c}{ Time } & Asthma & Control & $p$ \\
\hline$n$ & 29 & 31 & \\
Baseline & & & \\
$\quad \mathrm{DI}^{*}$ & $56(6)$ & $58(7)$ & 0.476 \\
$\quad \Delta \mathrm{RRS}_{\mathrm{DI}}(\mathrm{hPa} \mathrm{s} / \mathrm{L}) \dagger$ & $-0.69(1.03)$ & $-0.40(0.66)$ & 0.197 \\
$5 \mathrm{~min}$ & & & \\
$\quad \mathrm{DI}$ & $56(7)$ & $59(6)$ & 0.147 \\
$\quad \Delta \mathrm{RRS}_{\mathrm{DI}}(\mathrm{hPa} \mathrm{s} / \mathrm{L})$ & $-1.46(2.08)$ & $-0.41(1.41)$ & 0.02 \\
$15 \min ^{\mathrm{DI}}$ & $56(6)$ & $59(7)$ & 0.119 \\
$\quad \Delta \mathrm{RRS}_{\mathrm{DI}}(\mathrm{hPa} \mathrm{s} / \mathrm{L})$ & $-0.24(0.90)$ & $-0.16(0.86)$ & 0.750 \\
\hline
\end{tabular}

$*$ Volume of DI as \% baseline FVC.

$\dagger$ Difference in RRsi between post- and predeep inhalation (see Methods).

excessive proportions of filtered RRS data $(n=2)$. Altogether, the response to DI could be documented in 29 children with asthma and 31 controls. Overall, the DI volume was similar in asthma and control at the different times of the study (Table 3). However, $\Delta R_{R S_{D I}}$ was not significantly different between groups at baseline but significantly more negative in asthma than control at $5 \min (p=0.02)$ but not at $15 \mathrm{~min}$ after exercise (Table 3 ).

Sensitivity of response to exercise at specificity $>0.90$. The parameter ranking by largest sensitivity at specificity $>0.90$ gave the following order: 0.53 for $24 \%$ increase in RRsi; 0.45 for $27 \%$ increase in RRse or $5 \%$ decrease in $\mathrm{FEV}_{1}$; and 0.38 for $\Delta \mathrm{RRS}_{\mathrm{DI}}=-1.7 \mathrm{hPa} \mathrm{s} / \mathrm{L}$.

\section{DISCUSSION}

To the best of our knowledge, this is the first case-control identification of EIB using the FOT versus spirometry in primary school children. The novel findings are the significant difference between asthma and control in RRS response to exercise and response to DI after exercise and improved sensitivity of RRsi versus $\mathrm{FEV}_{1}$ as endpoint to the bronchomotor response.

The magnitude of the airway response to exercise in asthmatics is primarily dependent on the degree of bronchial inflammation (1), which has been shown to fluctuate with time (18) so that not all asthmatics present with EIB at time of referral (19). Exercise challenge performed at a time where bronchial inflammation is decreased would be less likely to be positive, and the test would, therefore, be associated with lower sensitivity. Because different lung function measurements describe different characteristics of airway obstruction (20), it is meaningful to question the diagnostic value of FOT with reference to spirometry, the usual endpoint to EIB testing. The optimal decision level for spirometry here was a 5\% decrease in $\mathrm{FEV}_{1}$, about twice the measured coefficient of repeatability. Although such amplitude of change relative to repeatability has previously been recommended to establish significant variation (21), the current threshold is smaller than the recommended $13 \%$. This threshold, associated with $63 \%$ sensitivity (3) and larger than the current findings at comparable specificity, was established in subjects aged $5-25$ y by comparing patients of this study (3) with controls from three different studies of the literature (22-24). Importantly, spirometry was obtained at earlier timings, i.e. starting 1 or $3 \mathrm{~min}$ after cessation of exercise $(3,22-24)$, and this could contribute to improve sensitivity because larger spirometric changes have been reported 3 min than 5 min after exercise (25). Asthmatics (3) and one control group (22) exercised on treadmill, whereas the other two healthy groups exercised by outdoor free run $(23,24)$. The free run may be associated with larger airway responses than for the treadmill (26), possibly contributing to raise the optimal threshold for diagnosis. In the current study, both asthmatics and controls were primary school children explored in the same technical environment, and it is worth noting that no healthy child showed a $>7 \%$ decrease in $\mathrm{FEV}_{1}$. The target heart rate of our exercise protocol was $80 \%$ of predicted maximal value, as recommended in adults rather than children $(2,16)$, and it is possible that the cutoff change in $\mathrm{FEV}_{1}$ could have been increased by aiming at 90\% predicted maximal heart rate (27). However, the current endpoint for heart rate was also used in another pediatric study and found satisfactorily to separate preschool children with asthma from those with nonspecific respiratory symptoms (25). Furthermore, the maximal heart rate actually achieved here was within the range reported in previous studies in children of comparable age $(3,23)$.

Besides the early description by Lenney and Milner (28), we are aware of only one case-control study in oscillation mechanics as endpoint to EIB in a pediatric population. The RRS was measured by the impulse oscillation technique in preschool children submitted to an outdoor free run (ambient temperature $-15^{\circ} \mathrm{C}$ to $+20^{\circ} \mathrm{C}$ ) and retested $2 \mathrm{~min}$ and later after exercise (29). The reported optimal decision level, a 30\% increase in mean RRS at $5 \mathrm{~Hz}$, was associated with a sensitivity of 0.62 at specificity $>0.90$ (29), both values being larger than in this report. Notable protocol variances could have explained this difference such as type of challenge and thermohygrometric environment $(2,26)$. Again, retesting at 5 min instead of 2 min may have contributed to the decrease in sensitivity by missing the early response. Conversely, early measurements may unfavorably impact on the FOT because the postexercise hyperventilation, which was mild here $5 \mathrm{~min}$ after exercise, is likely to decrease the signal-to-noise ratio and increase the RRS through its flow-dependent component (30). Although the aim of this study was not to assess the validity of different FOT techniques, it may also be wondered how much of the findings depend on the way the respiratory system is excited. The 
upper airway wall motion has been shown to induce underestimation of RRS and RRS response to challenge, particularly in children $(31,32)$ but was minimized here with the head generator (13). Because of the negative frequency dependence of the upper airway wall impedance, this artifact would have less impact at a lower frequency such as $5 \mathrm{~Hz}$, than at $12 \mathrm{~Hz}$ when pressure is varied directly at the mouth (12). Significant potentials of the "shunt-free" higher frequency RRS are the decreased likelihood of breathing versus signal flow harmonics crosstalk and improved time resolution and ability to identify time-dependent properties of airway mechanics. An alternative to the head generator would be the assessment of airway response to exercise by computing the respiratory admittance, another way to minimize the upper airway artifact during challenge $(33,34)$. Computing RRS as RRsi and RRse seemed advantageous, in view of the current observation that RRse exhibited less significant change after exercise and, in asthmatics, larger variability than RRsi, in keeping with previous reports (30). Altogether, we are unaware of real-life data comparing the impact of these different aspects of the FOT on the routine identification of EIB, but the issue certainly deserves further investigation.

This protocol entailed a number of full inspirations before exercising, including those needed for spirometry, which could have induced some degree of bronchoprotection, which has been defined as DI preventing or markedly reducing subsequently induced bronchoconstriction $(10,35)$. The contribution in this study is unclear because the phenomenon has been demonstrated in healthy adults taking deep breaths before histamine or methacholine challenge, is debated in asthma (36), and seems lacking in allergic subjects challenged with inhaled allergen (37). We are unaware of any systematic study of DI protecting from EIB, but it is worth noting that in subjects with an asthma attack, bronchodilation that occurs during a short exercising period (38) is followed by bronchoconstriction on prolongation of the effort (39).

The mechanisms accounting for the observed power difference between $\mathrm{FEV}_{1}$ and RRs may relate to the former being determined by expiratory flow limitation, whereas, at the oscillation frequency of interest, the latter is more dependent on central airways mechanics (20). In addition, spirometry requires inspiring to total lung capacity that stretches the intrathoracic airway wall, transiently dilating the lumen (40). As the effect is larger with mildly heightened bronchomotor tone (10), the estimate of EIB based on spirometry in asthma could be attenuated by the full inspiration, minimizing the difference to control, in contrast to measurements during tidal breathing. Indeed, the bronchodilation after the DI in asthma is supported by the significantly more negative $\Delta \mathrm{R}_{\mathrm{RS}}$ DI versus controls 5 min after exercise (Table 3). Furthermore, the repetition of DIs after exercise could possibly have damped amplitude of both spirometry and FOT estimates of the bronchoconstriction in asthmatics, with little effect in controls, contributing to lower the sensitivity compared with reports from the literature $(3,29)$.

The potential of $\Delta \mathrm{RRS}_{\mathrm{DI}}$ in detecting EIB was reduced by the significant proportion of rejected tests. Although the amplitude of the full inspiration was comparable between groups and reasonably reproducible at different times of the study in the selected subjects (Table 3), a major issue was the difficulty for some properly to achieve a breathing pattern regular enough throughout the maneuver. For instance, excessive salivation on prolonged contact with the mouthpiece induced swallowing, glottis closure, and irregular breathing. Transient shallow breathing after the DI was another reason for discarding the data. The time dependence of airway resistance in fact had been anticipated to be a possible limitation of RRS as a tool to assess the bronchomotor effects of DI (40). The impact of tidal airflow on RRS (Fig. 1) also stresses the importance of restricting such measurement to periods of regular breathing. Using end-inspiratory values may theoretically decrease the contribution of flow to RRsi, yet, transition from RRsi to RRse would have to be smooth enough to accurately identify undistorted near zero flow values (14). That the $\Delta \mathrm{RRS}_{\mathrm{DI}}$ could not be recovered in a significant proportion of subjects may induce a bias in interpreting the case-control difference, for instance, expressing the fact that asthmatic children may show lower RRs repeatability (41), but the overall proportion of data rejected was very similar in asthma and controls. Moreover, it may be argued that some sensitization of the $\Delta \mathrm{RRS}_{\mathrm{DI}}$ might have resulted from taking a through instead of mean RRsi after the DI, but the difference between asthma and control was significant after exercise and not at baseline. Although of limited practical value, in the current form, to identify the airway response to exercise, the DI maneuver seemed informative from a mechanistic point of view. Indeed, the case-control difference suggests increased bronchial wall hysteresis, and therefore smooth muscle contraction, as mechanism to the EIB. Healthy children may exhibit small degree of airway narrowing after exercise not associated with dilatory effect of DI, and the mechanism put forward was transient hyperemia in the airway wall (17) based on the experimental report that rapid fluid loading of the latter promotes airway narrowing insensitive to DI (42).

It is concluded that the endpoint based on RRsi seems beneficial in improving sensitivity of the EIB with reference to spirometry. An approximately $25 \%$ increase in Rrsi would provide the most specific indicator in the current conditions of the challenge. The threshold values disclosed here may require further evaluation using commercially available FOT systems with simplified protocols. The response to DI, as currently studied, does not improve sensitivity but may be of interest in highlighting the mechanism of airway obstruction, i.e. airway smooth muscle contraction in asthma and airway wall hyperemia in control.

Acknowledgments. We thank Dr René Peslin for valuable comments, Prof John Widdicombe for help with editing the article, B. Demoulin, C. Bonabel-Choné, and S. Méline for technical assistance, children and their parents, teachers, and administrators of public schools "Louis Pergaud, Laxou" and "Brabois Vandoeuvre." Dr René Peslin passed away while the article was finally processed. The authors express their condolences to his family. 


\section{REFERENCES}

1. Anderson SD 2002 Exercise-induced asthma in children: a marker of airway inflammation. Med J Aust 177:S61-S63

2. Anderson SD, Brannan JD 2003 Methods for "indirect" challenge tests including exercise, eucapnic voluntary hyperpnea, and hypertonic aerosols. Clin Rev Allergy Immunol 24:27-54

3. Godfrey S, Springer C, Bar-Yishay E, Avital A 1999 Cut-off points defining normal and asthmatic bronchial reactivity to exercise and inhalation challenges in children and young adults. Eur Respir J 14:659-668

4. Peslin R, Ying Y, Gallina C, Duvivier C 1992 Within-breath variations of forced oscillation resistance in healthy subjects. Eur Respir J 5:86-92

5. Collett PW, Brancatisano T, Engel LA 1983 Changes in the glottic aperture during bronchial asthma. Am Rev Respir Dis 128:719-723

6. Higenbottam T 1980 Narrowing of glottis opening in humans associated with experimentally induced bronchoconstriction. J Appl Physiol 49:403-407

7. Schweitzer C, Moreau-Colson C, Marchal F 2002 Respiratory impedance response to a deep inhalation in asthmatic children with spontaneous airway obstruction. Eur Respir J 19:1020-1025

8. Marchal F, Schweitzer C, Moreau-Colson C 2002 Respiratory impedance response to a deep inhalation in children with history of cough or asthma. Pediatr Pulmonol 33:411-418

9. Marchal F, Schweitzer C, Khallouf S 2003 Respiratory conductance response to a deep inhalation in children with exercise-induced bronchoconstriction. Respir Med 97:921-927

10. Brusasco V, Pellegrino R 2003 Complexity of factors modulating airway narrowing in vivo: relevance to assessment of airway hyperresponsiveness. J Appl Physiol 95:1305-1313

11. Knudson RJ, Lebowitz MD, Holberg CJ, Burrows B 1983 Changes in the normal maximal expiratory flow-volume curve with growth and aging. Am Rev Respir Dis 127:725-734

12. Oostveen E, MacLeod D, Lorino H, Farre R, Hantos Z, Desager K, Marchal F 2003 The forced oscillation technique in clinical practice: methodology, recommendations and future developments. Eur Respir J 22:1026-1041

13. Peslin R, Duvivier C, Didelon J, Gallina C 1985 Respiratory impedance measured with head generator to minimize upper airway shunt. J Appl Physiol 59:1790-1795

14. Marchal F, Schweitzer C, Demoulin B, Chone C, Peslin R 2004 Filtering artefacts in measurements of forced oscillation respiratory impedance in young children. Physiol Meas 25:1153-1166

15. Schweitzer C, Vu LT, Nguyen YT, Chone C, Demoulin B, Marchal F 2006 Estimation of the bronchodilatory effect of deep inhalation after a free run in children. Eur Respir J 28:89-95

16. Joos GF, O'Connor B, Anderson SD, Chung F, Cockcroft DW, Dahlen B, DiMaria G, Foresi A, Hargreave FE, Holgate ST, Inman M, Lotvall J, Magnussen H, Polosa R, Postma DS, Riedler J 2003 Indirect airway challenges. Eur Respir J 21:10501068

17. Marchal F, Schweitzer C, Werts F, Vu LT, DeMoulin B, Nguyen YT 2008 Post-exercise airway narrowing in healthy primary school children. Pediatr Res 63:328-331

18. Ward C, Reid DW, Orsida BE, Feltis B, Ryan VA, Johns DP, Walters EH 2005 Inter-relationships between airway inflammation, reticular basement membrane thickening and bronchial hyper-reactivity to methacholine in asthma; a systematic bronchoalveolar lavage and airway biopsy analysis. Clin Exp Allergy 35:1565-1571

19. Carlsen KH, Engh G, Mork M 2000 Exercise-induced bronchoconstriction depends on exercise load. Respir Med 94:750-755

20. Marchal F, Schweitzer C, Thuy LV 2005 Forced oscillations, interrupter technique and body plethysmography in the preschool child. Paediatr Respir Rev 6:278-284

21. Sly PD, Lanteri CJ, Raven JM 1991 Do wheezy infants recovering from bronchiolitis respond to inhaled salbutamol? Pediatr Pulmonol 10:36-39
22. Backer V, Bach-Mortensen N, Dirksen A 1989 Prevalence and predictors of bronchial hyperresponsiveness in children aged 7-16 years. Allergy 44:214-219

23. Haby MM, Peat JK, Mellis CM, Anderson SD, Woolcock AJ 1995 An exercise challenge for epidemiological studies of childhood asthma: validity and repeatability. Eur Respir J 8:729-736

24. Riedler J, Reade T, Dalton M, Holst D, Robertson C 1994 Hypertonic saline challenge in an epidemiologic survey of asthma in children. Am J Respir Crit Care Med 150:1632-1639

25. Vilozni D, Bentur L, Efrati O, Barak A, Szeinberg A, Shoseyov D, Yahav Y, Augarten A 2007 Exercise challenge test in 3- to 6-year-old asthmatic children. Chest 132:497-503

26. Anderson SD, Connolly NM, Godfrey S 1971 Comparison of bronchoconstriction induced by cycling and running. Thorax 26:396-401

27. Carlsen KH, Carlsen KC 2002 Exercise-induced asthma. Paediatr Respir Rev 3:154-160

28. Lenney W, Milner AD 1978 Recurrent wheezing in the preschool child. Arch Dis Child 53:468-473

29. Malmberg LP, Makela MJ, Mattila PS, Hammaren-Malmi S, Pelkonen AS 2008 Exercise-induced changes in respiratory impedance in young wheezy children and nonatopic controls. Pediatr Pulmonol 43:538-544

30. Schweitzer C, Chone C, Marchal F 2003 Influence of data filtering on reliability of respiratory impedance and derived parameters in children. Pediatr Pulmonol 36:502508

31. Marchal F, Mazurek H, Habib M, Duvivier C, Derelle J, Peslin R 1994 Input respiratory impedance to estimate airway hyperreactivity in children: standard method versus head generator. Eur Respir J 7:601-607

32. Cauberghs M, Van de Woestijne KP 1989 Effect of upper airway shunt and series properties on respiratory impedance measurements. J Appl Physiol 66:2274-2279

33. Farré R, Rotger M, Marchal F, Peslin R, Navajas D 1999 Assessment of bronchial reactivity by forced oscillation admittance avoids the upper airway artefact. Eur Respir J 13:761-766

34. Nguyen YT, Demoulin B, Schweitzer C, Bonabel-Chone C, Marchal F 2007 Identification of bronchodilator responsiveness by forced oscillation admittance in children. Pediatr Res 62:348-352

35. Cockcroft DW, Davis BE 2006 The bronchoprotective effect of inhaling methacholine by using total lung capacity inspirations has a marked influence on the interpretation of the test result. J Allergy Clin Immunol 117:1244-1248

36. Skloot G, Togias A 2003 Bronchodilation and bronchoprotection by deep inspiration and their relationship to bronchial hyperresponsiveness. Clin Rev Allergy Immunol 24:55-72

37. Pyrgos G, Kapsali T, Permutt S, Togias A 2003 Absence of deep inspiration-induced bronchoprotection against inhaled allergen. Am J Respir Crit Care Med 167:16601663

38. Crimi E, Pellegrino R, Smeraldi A, Brusasco V 2002 Exercise-induced bronchodilation in natural and induced asthma: effects on ventilatory response and performance. J Appl Physiol 92:2353-2360

39. Milanese M, Saporiti R, Bartolini S, Pellegrino R, Baroffio M, Brusasco V, Crimi E 2009 Bronchodilator effects of exercise hyperpnea and albuterol in mild-to-moderate asthma. J Appl Physiol 107:494-499

40. Pellegrino R, Sterk PJ, Sont JK, Brusasco V 1998 Assessing the effect of deep inhalation on airway calibre: a novel approach to lung function in bronchial asthma and COPD. Eur Respir J 12:1219-1227

41. Lall CA, Cheng N, Hernandez P, Pianosi PT, Dali Z, Abouzied A, Maksym GN 2007 Airway resistance variability and response to bronchodilator in children with asthma. Eur Respir J 30:260-268

42. Pellegrino R, Dellaca R, Macklem PT, Aliverti A, Bertini S, Lotti P, Agostoni P, Locatelli A, Brusasco V 2003 Effects of rapid saline infusion on lung mechanics and airway responsiveness in humans. J Appl Physiol 95:728-734 